\begin{tabular}{|c|c|}
\hline Title & Finite element analysis of multi-turn magnetoplated coils using homogenization method \\
\hline Author(s) & Fujita, Shogo; Igarashi, Hajime \\
\hline Citation & $\begin{array}{l}\text { Electrical engineering in Japan, 205(4), 23-31 } \\
\text { https://doi.org/10.1002/ej. } 23156\end{array}$ \\
\hline Issue Date & 201812 \\
\hline Doc URL & http:/hdl.handle.net/2115/76237 \\
\hline Rights & $\begin{array}{l}\text { This is the peer reviewed version of the following article: Electrical engineering in Japan, } V \text { olume205(4)(2018), 23-31, } \\
\text { which has been published in final form at https://doi.org/10.1002/eej.23156. This article may be used for non- } \\
\text { commercial purposes in accordance with Wiley Terms and Conditions for U se of Self-A rchived Versions. }\end{array}$ \\
\hline Type & article (author version) \\
\hline File Information & manuscript_HUSCUP.pdf \\
\hline
\end{tabular}

Instructions for use 


\title{
Finite element analysis of multi-turn magnetoplated coils using homogenization method
}

\author{
Shogo Fujita | Hajime Igarashi PhD
}

Applied Electromagnetics Laboratory, Research Group of Informatics for Systems Synthesis, Division of Systems Science and Informatics, Graduate School of Information Science and Technology, Hokkaido University, Sapporo, Japan

\section{Correspondence}

Shogo Fujita, Applied Electromagnetics Laboratory, Research Group of Informatics for Systems Synthesis, Division of Systems Science and Informatics, Graduate School of Information Science and Technology, Hokkaido University, Sapporo, Hokkaido 060-0808, Japan.

Email: fujita@em-si.eng.hokudai.ac.jp

Translated from Volume 137 Number 3, pages 223-229, DOI: 10.1541/ieejpes.137.223 of IEEJ Transactions on Power and Energy (Denki Gakkai Ronbunshi B)

\begin{abstract}
Magnetoplated wires (MPW) are effective to reduce eddy current losses in power devices such as converters and contactless power transfer systems. In the conventional finite element analysis (FEA) of MPW, elements must be sufficiently smaller than the skin depth. This results in large number of unknowns. This article proposes a homogenization method using complex permeability for FEA of magnetoplated coils so as to circumvent this problem. The coil impedances obtained by the proposed method with coarse elements are shown to agree well with those computed in the conventional FEA with fine elements.
\end{abstract}

\section{K E Y W O R D S}

eddy current, finite element method, homogenization, magnetoplated wire, proximity effect, skin effect

\section{1 | INTRODUCTION}

Magnetoplated wires (MPW) are copper wires plated with a magnetic material. Due to such structure, magnetic flux penetrating in the copper wire can be reduced, which leads to suppression of eddy currents (ECs) caused by proximity effect. Thus, the use of MPW is considered in magnetoplated (MP) coils for converters, transformers, and contactless power transfer systems ${ }^{1-4}$ as well as in EC displacement sensors. ${ }^{5}$

When applying finite element analysis (FEA) to electric devices, a coil must be divided into finite elements (FEs) in order to involve ECs in analysis. Particularly, skin effect and proximity effect become pronounced as driving frequency is increased, and these effects must be taken into account in element division. When considering ECs in devices with MP coils, an enormous number of FEs are needed to discretize such coils. This is especially true for $3 \mathrm{D}$ analysis when the number of unknowns in FEA is extremely large, and computation takes a long time.

In order to avoid fine element division caused by multiscale structure, we proposed a homogenization method to analyze ECs in coils. ${ }^{6,7}$ In this method, complex effective permeability is set in coil region with regard to demagnetizing field effect due to EC caused by proximity effect, and coils are handled as macro objects. Thus losses, coil inductance, and other parameters can be determined at almost same accuracy as in conventional quasi-static electromagnetic field analysis, without fine element division. It was also shown that the proposed method offers good agreement with measured values of coil ac resistance. ${ }^{7}$ However, no FEA using the homogenization method has been yet proposed for MP coils.

In this article, we propose a homogenized FEA for MP coils. In so doing, complex effective permeability of MP coil placed in uniform ac magnetic field is determined analytically, and used in combination with Ollendorff's equation ${ }^{8}$ to obtain macro permeability. We apply the proposed method to MP coil model, and compare results with those of conventional FEA. Then we discuss optimization of MP coil plating thickness using this method.

\section{I SKIN EFFECT AND PROXIMITY EFFECT IN ROUND MPW}

\section{1 | Electromagnetic field in round MPW}

Variables used in this article are listed in Table 1. Actually, plating of MPW has some electrical conductivity but 
T A B L E 1 Nomenclature

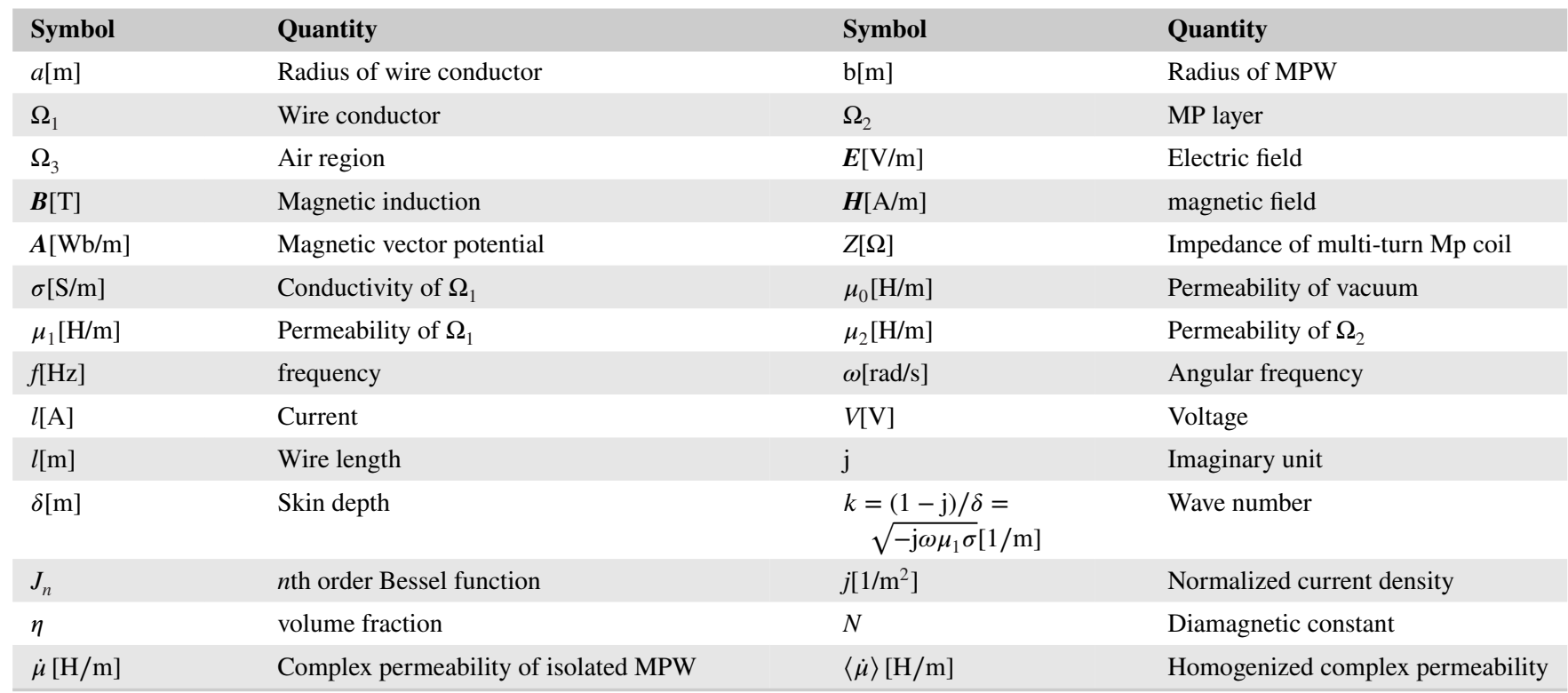

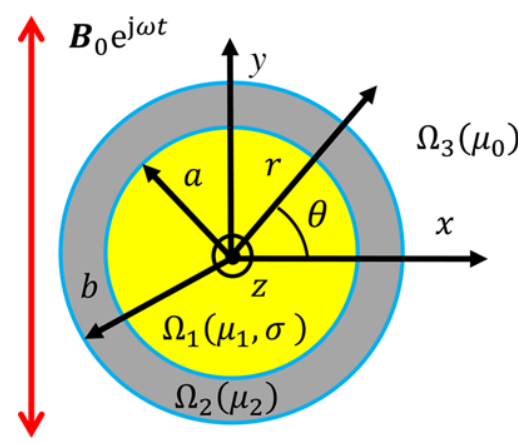

F I G U R E 1 Cross-section of an MPW immersed in uniform, time-harmonic magnetic field [Color figure can be viewed at wileyonlinelibrary.com]

in this article, we assume for simplicity that magnetic plating is an insulator. Besides, we ignore longitudinal curvature and assume a straight conductor. As shown in Figure 1, we introduce cylindrical coordinates $(r, \theta, z)$ so that current flows along $z$-axis, and uniform ac magnetic field $\boldsymbol{B}_{0} \mathrm{e}^{\mathrm{j} w t}$ is applied along $y$-axis. Because the system is symmetrical, electric field inside and outside the MPW can be expressed as $\boldsymbol{E}=E_{z}(r, \theta) \boldsymbol{i}_{z}$.

We consider the following quasi-static approximated Maxwell's equations in frequency domain as the governing equations for electromagnetic field inside and outside the MPW:

$$
\begin{aligned}
\operatorname{rot} \boldsymbol{H} & =\sigma \boldsymbol{E}, \\
\operatorname{rot} \boldsymbol{E} & =-j \omega \mu \boldsymbol{H} .
\end{aligned}
$$

Below we find electromagnetic field inside and outside the MPW by solving Equation (1a) for each region. First, with regard to $\Omega_{1}$, Equation (1) reduces to the following Helmholtz's equation:

$$
\frac{\partial^{2} E_{z}}{\partial r^{2}}+\frac{1}{r} \frac{\partial E_{z}}{\partial r}+\frac{1}{r^{2}} \frac{\partial^{2} E_{z}}{\partial \theta^{2}}+k^{2} E_{z}=0
$$

Here $k$ is wave number (see Table 1). Now, the following ordinary differential equations are obtained by applying separation of variables $E_{z}(r, \theta)=\mathrm{R}(\mathrm{r}) \Theta(\theta)$ to Equation (2):

$$
\begin{aligned}
& r^{2} R^{\prime \prime}(r)+r R^{\prime}(r)+\left(k^{2} r^{2}-\beta^{2}\right) R(r)=0, \\
& \Theta^{\prime \prime}(\theta)+\beta^{2} \Theta(\theta)=0 .
\end{aligned}
$$

Here $\beta$ is separation constant. Under uniform ac magnetic field, a magnetic dipole appears on the wire cross section due to EC. In so doing, electric field is composed of component independent of $\theta$ (including skin effect) and component produced by external magnetic field (related to proximity effect). Corresponding solution to Equation (3) is as follows ${ }^{6,7}$ :

$$
E_{z}(r, \theta)=p_{0} J_{0}(k r)+p_{1} J_{1}(k r) \cos \theta .
$$

Here $J_{n}$ is $n$th order Bessel function, and $p_{0}$ and $p_{1}$ are constants. The first and second terms in Equation (4a) pertain, respectively, to electric field representing skin effect and dipole electric field representing proximity effect. Magnetic field in $\Omega_{1}$ is obtained by substituting Equation (4a) into Equation (1b) as shown below:

$$
H_{\theta}(r, \theta)=\frac{j k}{\omega \mu_{1}}\left\{p_{0} J_{1}(k r)-p_{1} J_{1}^{\prime}(k r) \cos \theta\right\}
$$


Next, regarding $\Omega_{2}$, magnetic plating is assumed as an insulator, and therefore, electric field is governed by the following Laplace's equation with $\sigma=0$ in Equation (2):

$$
\frac{\partial^{2} E_{z}}{\partial r^{2}}+\frac{1}{r} \frac{\partial E_{z}}{\partial r}+\frac{1}{r^{2}} \frac{\partial^{2} E_{z}}{\partial \theta^{2}}=0
$$

Now consider the following dipole and uniform magnetic field components as a solution to Equation (5):

$$
E_{z}(r, \theta)=\left(\frac{q_{1}}{r}+\mathrm{j} \omega \alpha B_{0} r\right) \cos \theta .
$$

In the above expression, $q_{1}$ and $\alpha$ are constants. Here we omit components independent of $\theta$ ( $\log r$ terms) as irrelevant to subsequent discussion. The first term in Equation (6a) pertains to magnetic dipole in copper wire, while the second term expresses component corresponding to ac magnetic field ( $\alpha$ represents magnetic shielding effect). Substituting Equation (6a) into Equation (1b) and introducing total current $I$ in copper wire, magnetic field in $\Omega_{2}$ can be found from Ampère's circuital integral law as shown below:

$$
\begin{aligned}
& H_{\theta}(r, \theta)=\frac{I}{2 \pi r}-\frac{\mathrm{j}}{\omega \mu_{2}}\left(-\frac{q_{1}}{r^{2}}+\mathrm{j} \omega \alpha B_{0}\right) \cos \theta, \\
& H_{r}(r, \theta)=-\frac{\mathrm{j}}{\omega \mu_{2}}\left(-\frac{q_{1}}{r^{2}}+\mathrm{j} \omega \alpha B_{0}\right) \sin \theta .
\end{aligned}
$$

Finally, regarding $\Omega_{3}$, electric and magnetic fields can be determined by solving Equation (5) same as with $\Omega_{2}$.

$$
\begin{aligned}
& E_{z}(r, \theta)=\left(\frac{q_{2}}{r}+\mathrm{j} \omega B_{0} r\right) \cos \theta \\
& H_{\theta}(r, \theta)=\frac{I}{2 \pi r}-\frac{\mathrm{j}}{\omega \mu_{2}}\left(-\frac{q_{2}}{r^{2}}+\mathrm{j} \omega B_{0}\right) \cos \theta
\end{aligned}
$$

Here $q_{2}$ is a constant. The first term in Equation (7a) pertains to magnetic moment of MPW as seen from outside.

Thus, electromagnetic field inside and outside MPW is expressed by Equations 4, 6, and 7. Next, we determine unknown constants $p_{0}, p_{1}, q_{1}, q_{2}$, and $\alpha$ included in these expressions. First, $p_{0}$ can be found from Ampère's circuital integral law as follows ${ }^{6,7}$ :

$$
p_{0}=\frac{k I}{2 \pi a \sigma J_{1}(\zeta)} .
$$

Here $\zeta=k a$. On the other hand, constants in terms including $\cos \theta$ are determined from the continuity of tangential components of electric and magnetic fields. First, the following holds true on the boundary $\Omega_{21}$ of $\Omega_{1}$ and $\Omega_{2}$ :

$$
\begin{gathered}
p_{1} J_{1}(\zeta)=\frac{q_{1}}{a}+j \omega \alpha B_{0} a, \\
-\frac{\mathrm{j} k}{\omega \mu_{1}} p_{1} J_{1}^{\prime}(\zeta)=\frac{j}{\omega \mu_{2}}\left(-\frac{q_{1}}{a}+\mathrm{j} \omega \alpha B_{0}\right) .
\end{gathered}
$$

Similarly, the following holds true on the boundary $\Omega_{32}$ of $\Omega_{2}$ and $\Omega_{3}$ :

$$
\begin{gathered}
\frac{q_{1}}{b}+\mathrm{j} \omega \alpha B_{0} b=\frac{q_{2}}{b}+\mathrm{j} \omega B_{0} b, \\
-\frac{\mathrm{j}}{\omega \mu_{2}}\left(-\frac{q_{1}}{b_{2}}+\mathrm{j} \omega \alpha B_{0}\right)=-\frac{\mathrm{j}}{\omega \mu_{0}}\left(-\frac{q_{2}}{b_{2}}+\mathrm{j} \omega B_{0}\right) .
\end{gathered}
$$

The following simultaneous equations are obtained from Equations (9) and (10):

$$
\begin{gathered}
{\left[\begin{array}{cccc}
J_{1} & -1 / a & -\mathrm{j} \omega a B_{0} & 0 \\
k v_{1} J_{1}^{\prime}(\zeta) & 1 / a^{2} & -\mathrm{j} \omega B_{0} & 0 \\
0 & 1 / b & \mathrm{j} \omega b B_{0} & -1 / b \\
0 & v_{2} / b^{2} & -\mathrm{j} \omega v_{2} B_{0} & -1 / b^{2}
\end{array}\right]\left[\begin{array}{l}
p_{1} \\
q_{1} \\
\alpha \\
q_{2}
\end{array}\right]} \\
=\left[\begin{array}{c}
0 \\
0 \\
\mathrm{j} \omega b B_{0} \\
-\mathrm{j} \omega b B_{0}
\end{array}\right] .
\end{gathered}
$$

Here $v_{1}=\mu_{2} / \mu_{1}, v_{2}=\mu_{0} / \mu_{2}$. The unknown constants can be found by solving Equation (11) as shown below:

$$
\begin{aligned}
{\left[\begin{array}{c}
p_{1} \\
q_{1} \\
\alpha \\
q_{2}
\end{array}\right] } & =\frac{a^{2} b^{3}}{\mathrm{j} \omega B_{0}\left(\lambda_{3} J_{1}(\zeta)+\lambda_{4} v_{1} \zeta J_{1}^{\prime}(\zeta)\right)} \\
& \times\left[\begin{array}{c}
2\left(\mathrm{j} \omega B_{0}\right)^{2}\left(J_{1}(\zeta)-v_{1} \zeta J_{1}^{\prime}(\zeta)\right) / b \\
-2 \mathrm{j} \omega B_{0}\left(J_{1}(\zeta)+v_{1} \zeta J_{1}^{\prime}(\zeta)\right) / a^{2} b \\
\left(\mathrm{j} \omega B_{0}\right)^{2}\left(\lambda_{1} J_{1}(\zeta)+\lambda_{2} v_{1} \zeta J_{1}^{\prime}(\zeta)\right) / a^{2} b
\end{array}\right] .
\end{aligned}
$$

Here,

$$
\begin{aligned}
& \lambda_{1}=-c_{1} v_{2}-c_{2}, \\
& \lambda_{2}=c_{2} v_{2}+c_{1}, \\
& \lambda_{3}=c_{1} v_{2}-c_{2}, \\
& \lambda_{4}=-c_{2} v_{2}+c_{1} .
\end{aligned}
$$

Here $c_{1}=a^{2}-b^{2}, c_{2}=a^{2}+b^{2}$. In case of no magnetic plating, $b \rightarrow a, \mu_{2} \rightarrow \mu_{0}$ in Equation (12), and solution reduces to the following ${ }^{6,7}$ :

$$
\left[\begin{array}{l}
p_{1} \\
q_{1}
\end{array}\right]=\frac{\mathrm{j} \omega a^{2} B_{0}}{J_{1}(\zeta)+v_{1} \zeta J_{1}^{\prime}(\zeta)}\left[\begin{array}{c}
2 / a \\
J_{1}(\zeta)-v_{1} \zeta J_{1}^{\prime}(\zeta)
\end{array}\right] .
$$

At the same time, $\alpha \rightarrow 1, q_{2} \rightarrow q_{1}$. 


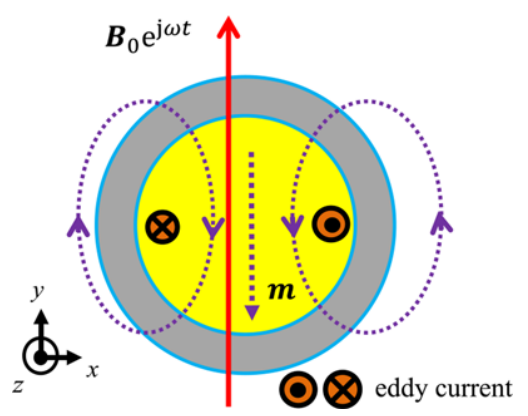

F I G U R E 2 Diamagnetic field caused by proximity effect [Color figure can be viewed at wileyonlinelibrary.com]

\subsection{Complex effective permeability of MPW}

With MPW placed in uniform magnetic field, magnetic flux is likely to concentrate within magnetic plating layer while a part of the flux passes through the wire, and EC flows in the copper wire so as to cancel this flux linkage (proximity effect). This EC produces diamagnetic field across the wire as shown in Figure 2 (in the diagram, $m$ denotes magnetic moment). Such EC forms dipole magnetic field in $\Omega_{3}$ as shown below ${ }^{6,7}$ :

$$
A_{z}=-\frac{\mu_{0}}{2 \pi} \frac{m}{r} \cos \theta
$$

Electric field generated outside MPW by magnetic dipole in copper wire is represented by the first term of Equation (7a). According to Equation (12a), the coefficient $q_{2}$ is determined as follows:

$$
\begin{gathered}
q_{2}=\mathrm{j} \omega B_{0} b^{2} \Lambda(\omega), \\
\Lambda(\omega)=\frac{\lambda_{1} J_{1}(\zeta)+\lambda_{2} v_{1} \zeta J_{1}^{\prime}(\zeta)}{\lambda_{3} J_{1}(\zeta)+\lambda_{4} v_{1} \zeta J_{1}^{\prime}(\zeta)} .
\end{gathered}
$$

Now, the following is obtained by substituting the first term of Equation (7a) and Equation (14) in $\boldsymbol{E}=-\mathrm{j} \omega \boldsymbol{A}$ :

$$
m=-\mathrm{j} \frac{2 \pi}{\mu_{0} \omega} q_{2} .
$$

Besides, in this case, magnetization in MPW cross section is $M=m /\left(\pi b^{2}\right)$; that is, the following is true:

$$
M=-\mathrm{j} \frac{2}{\mu_{0} \omega b^{2}} q_{2} .
$$

By substituting Equation (15) into Equation (17), frequency characteristic of $M$ can be expressed as follows:

$$
M=2 \frac{B_{0}}{\mu_{0}} \Lambda(\omega) .
$$

At the limit of static magnetic field $\omega \rightarrow 0$, Equation (18) becomes as follows:

$$
M=2 \frac{B_{0}}{\mu_{0}} \frac{\lambda_{1}+v_{1} \lambda_{2}}{\mu_{0} \lambda_{3}+v_{1} \lambda_{4}} .
$$

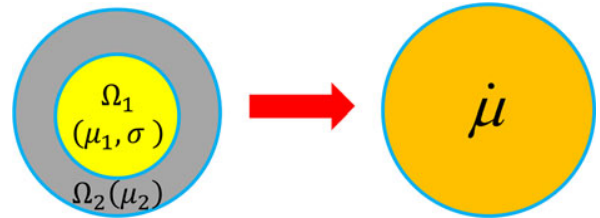

F I G U RE 3 Homogenization of MPW [Color figure can be viewed at wileyonlinelibrary.com]

Besides, without magnetic plating, Equation (19) reduces to the following well-known expression for cylinder magnetization in uniform field (demagnetization factor: 1/2):

$$
M=2 \frac{B_{0}}{\mu_{0}} \frac{\mu_{1}-\mu_{0}}{\mu_{1}+\mu_{0}} .
$$

Now we define MPW complex effective permeability $\dot{\mu}$ so that MPW magnetization in Equation (18) can be written in the form of Equation (20) at arbitrary frequency. In so doing, Equation (18) becomes as follows:

$$
M=2 \frac{B_{0}}{\mu_{0}} \frac{\dot{\mu}-\mu_{0}}{\dot{\mu}+\mu_{0}} .
$$

Therefore,

$$
2 \frac{B_{0}}{\mu_{0}} \Lambda(\omega)=2 \frac{B_{0}}{\mu_{0}} \frac{\dot{\mu}-\mu_{0}}{\dot{\mu}+\mu_{0}} .
$$

The following is obtained from Equation (22):

$$
\dot{\mu}=\mu_{0} \frac{1+\Lambda(\omega)}{1-\Lambda(\omega)} .
$$

Without magnetic plating, Equation (23) reduces to complex effective permeability of non-plated conductor as shown below 6,7 .

$$
\dot{\mu}=\mu_{1} \frac{J_{1}(\zeta)}{\zeta J_{0}(\zeta)-J_{1}(\zeta)} .
$$

Besides, at the limit of static magnetic field $\omega \rightarrow 0$, Equation (24) converges to $\mu_{1}$ and coincides with relative permeability of the wire.

Using $\dot{\mu}$, MPW can be homogenized as shown in Figure 3. In this case, complex power $P_{\text {prox }}$ related to proximity effect can be estimated as follows:

$$
\begin{aligned}
P_{\mathrm{prox}} & =\frac{\mathrm{j} \omega}{2} \int_{\Omega_{c}} \dot{\mu}|\boldsymbol{H}|^{2} d v \\
& =\mathrm{j} \omega \pi b^{2} l \frac{\dot{\mu}}{2}\left|\frac{2}{\dot{\mu}+\mu_{0}}\right|^{2} B_{0}^{2} \\
& =\mathrm{j} \omega \pi b^{2} l \frac{B_{0}^{2}}{2 \mu_{0}}\left\{2 \operatorname{Im}(\Lambda(\omega))-|\Lambda(\omega)|^{2}+1\right\} .
\end{aligned}
$$


Besides, in case of multi-turn MP coil, homogenization of cross section including MPW and air gap is effective. Such homogenized macro complex effective permeability can be obtained by substituting Equation (23) into the following Ollendorff's equation ${ }^{6,7}$ :

$$
\langle\dot{\mu}\rangle=\mu_{0}\left\{1+\frac{\eta\left(\dot{\mu}_{r}-1\right)}{1+N(1-\eta)\left(\dot{\mu}_{r}-1\right)}\right\} .
$$

Equation (26) is known to be essentially equivalent to Clausius-Mossotti formula used in calculation of macro dielectric constant as well as to Maxwell-Garnett formula used in high-frequency electromagnetic field analysis. ${ }^{7}$

\section{3 | Energy conservation law}

In this subsection, we consider MPW power. From Equation (1), energy conservation law is obtained as shown below (the first term on the right side denotes power related to proximity effect):

$$
\begin{aligned}
& \frac{1}{2} \int_{\Omega_{1}}\left(\sigma|\boldsymbol{E}|^{2}+\mathrm{j} \omega \mu_{1}|\boldsymbol{H}|^{2}\right) d v+\frac{\mathrm{j} \omega}{2} \int_{\Omega_{2}} \mu_{2}|\boldsymbol{H}|^{2} d v \\
& =-\frac{1}{2} \int_{\partial \Omega 32} \boldsymbol{E} \times \boldsymbol{H}^{*} \cdot d \boldsymbol{S}+P_{\text {skin }}+P_{\mathrm{mp}}
\end{aligned}
$$

On the right side of Equation (27), the first term pertains to power related to magnetic dipole in copper wire (i.e., power related to proximity effect), $P_{\text {skin }}$ is power due to skin effect, and $P_{\mathrm{mp}}$ expresses increase of magnetic energy accumulated in magnetic plating (the second and third terms on the right side of Equation (27) are not needed if electromagnetic field in the first term includes component produced by circulating current). Besides, power supplied from power source is equal to increase of magnetic energy inside and outside MPW and joule loss inside conductor; therefore, the following is true (9):

$$
\frac{V I^{*}}{2}=\frac{\mathrm{j} \omega}{2} \int_{\Omega} \mu|\boldsymbol{H}|^{2} d v+\frac{1}{2} \int_{\Omega_{1}} \sigma|\boldsymbol{E}|^{2} d v
$$

Here $\Omega=\Omega_{1}+\Omega_{2}+\Omega_{3}$. The following is obtained from Equations (27) and (28):

$$
\begin{aligned}
\frac{V I^{*}}{2}= & \frac{\mathrm{j} \omega}{2} \int_{\Omega_{3}} \mu_{0}|\boldsymbol{H}|^{2} d v \\
& -\frac{1}{2} \int_{\sigma \Omega_{32}} \boldsymbol{E} \times \boldsymbol{H}^{*} \cdot d \boldsymbol{S}+P_{\mathrm{skin}}+P_{\mathrm{mp}}
\end{aligned}
$$

The second term on the right side of Equation (29) can be expressed in the following way using Equation (7):

$$
\begin{aligned}
& -\frac{1}{2} \int_{\sigma \Omega_{32}} \boldsymbol{E} \times \boldsymbol{H}^{*} \cdot d \boldsymbol{S} \\
& =\mathrm{j} \omega \pi b^{2} l \frac{B_{0}^{2}}{2 \mu_{0}}\left\{2 \operatorname{Im}(\Lambda(\omega))-|\Lambda(\omega)|^{2}+1\right\} .
\end{aligned}
$$

Equation (30) coincides with complex power $P_{\text {prox }}$ due to proximity effect obtained from complex effective permeability (Equation (25)). Therefore, power related to proximity effect can be properly estimated when MP coil cross section is homogenized using Equations (23) and (26).

On the other hand, power in copper wire region $\Omega_{1}$ can be obtained by integrating Poynting vector over $\partial \Omega_{21}$. Thus, the following is obtained from Equation (4):

$$
\begin{aligned}
& -\frac{1}{2} \int_{\partial \Omega_{21}} \boldsymbol{E} \times \boldsymbol{H}^{*} \cdot d \boldsymbol{S} \\
& \quad=\frac{R_{0} \zeta J_{0}(\zeta)}{4 J_{1}(\zeta)}|I|^{2}-\mathrm{j} \frac{l \pi(\zeta)^{*}}{2 \omega \mu_{1}}\left|p_{1}\right|^{2} J_{1}(\zeta)\left(J_{1}^{\prime}(\zeta)\right)^{*}
\end{aligned}
$$

Here $R_{0}=1 /\left(\sigma \pi a^{2}\right)$ denotes dc resistance. The second term of Equation (31) is related to proximity effect; this term is included in Equation (30). Therefore, $P_{\text {skin }}$ is expressed by the first term on the right side. Besides, increase of magnetic energy in magnetic plating region $\Omega_{2}$ can be estimated as shown below:

$$
\begin{aligned}
& \frac{j \omega}{2} \int_{\Omega_{2}} \mu|\boldsymbol{H}|^{2} d v \\
& =\mathrm{j} \omega \mu_{2} l \pi\left|\frac{I}{2 \pi}\right|^{2} \log \left(\frac{b}{a}\right)+\mathrm{j} \frac{l \pi}{2 \omega \mu_{2}} \\
& \quad \times\left\{\frac{q_{1}^{2}}{2}\left(\frac{1}{b^{2}}-\frac{1}{a^{2}}\right)+\mathrm{j} 2 \omega \mathrm{q}_{1} \alpha B_{0} \log \left(\frac{b}{a}\right)\right. \\
& \left.\quad+\frac{\omega^{2} \alpha^{2} B_{0}^{2}}{2}\left(b^{2}-a^{2}\right)\right\} .
\end{aligned}
$$

The second and third terms in Equation (32) pertain to magnetic dipole; these are also included in Equation (30). Therefore, $P_{\mathrm{mp}}$ is the first term on the right side of Equation (32).

From the above, total power can be expressed as follows:

$$
\begin{aligned}
\frac{V I^{*}}{2}= & \frac{\mathrm{j} \omega}{2} \int_{\Omega} \mu|\boldsymbol{H}|^{2} d v+\frac{R_{0} \zeta J_{0}(\zeta)}{4 J_{1}(\zeta)}|I|^{2} \\
& +\mathrm{j} \omega \mu_{2} l \pi\left|\frac{I}{2 \pi}\right|^{2} \log \left(\frac{b}{a}\right) .
\end{aligned}
$$


Here we assume that in MP coil region, permeability in the first term on the right side is $\langle\dot{\mu}\rangle$ expressed by Equations (23) and (26). The first term on the right side of Equation (33) is power due to proximity effect, the second term is power due to skin effect, and the third term is increase of magnetic energy accumulated in magnetic plating layer due to input current. Proximity effect and skin effect are orthogonal to each other; hence no interference terms appear. ${ }^{6,7,10}$ Without magnetic plating, Equation (33) becomes as shown below, that is, reduces to power in conventional copper coil:

$$
\frac{V I^{*}}{2}=\frac{\mathrm{j} \omega}{2} \int_{\Omega} \mu|\boldsymbol{H}|^{2} d v+\frac{R_{0} \zeta J_{0}(\zeta)}{4 J_{1}(\zeta)}|I|^{2} .
$$

Besides, assuming the following at sufficient distance from coil:

$$
\mathrm{j} \omega \boldsymbol{A} \times \boldsymbol{H}=0 .
$$

Equation (33) can be expressed using magnetic vector potential as shown below:

$$
V=\mathrm{j} \omega \int_{\Omega_{c}} \boldsymbol{A} \cdot \mathbf{j}^{*} d v+\frac{R_{0} \zeta J_{0}(\zeta)}{2 J_{1}(\zeta)} I+\mathrm{j} \omega \mu_{2} l \frac{I}{2 \pi} \log \left(\frac{b}{a}\right) .
$$

Here $\Omega_{\mathrm{c}}=\Omega_{1}+\Omega_{2}$, and, $\mathbf{j}\left[1 / \mathrm{m}^{2}\right]$ denotes current density in case that unit current flows in a single copper wire; in case of current $I$, the current density is $\mathbf{J}=I \mathbf{j}$. Now, magnetic vector potential in Equation (36) can be obtained by FE method.

\section{3 | COMPARISON WITH QUASI- STATIC ELECTROMAGNETIC FIELD ANALYSIS}

\subsection{Complex effective permeability of MP coil}

First, we consider frequency characteristic of macro complex effective relative permeability $\left\langle\dot{\mu}_{r}\right\rangle=\langle\dot{\mu}\rangle / \mu_{0}$ of MP coil obtained in Section 2. By way of example, consider 81-turn MP coil as shown in Figure 4. Figure 5 shows frequency char-

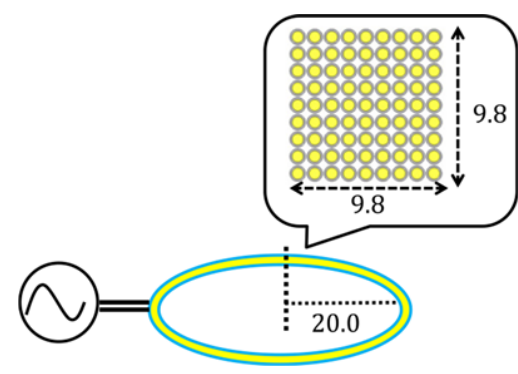

F I G U R E 4 Eighty-one-turn MP coil (unit: mm) [Color figure can be viewed at wileyonlinelibrary.com]

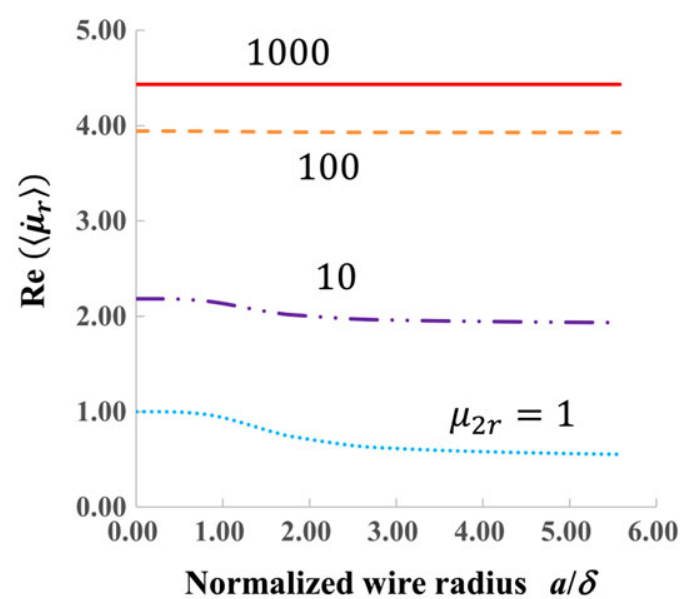

(a) Real part

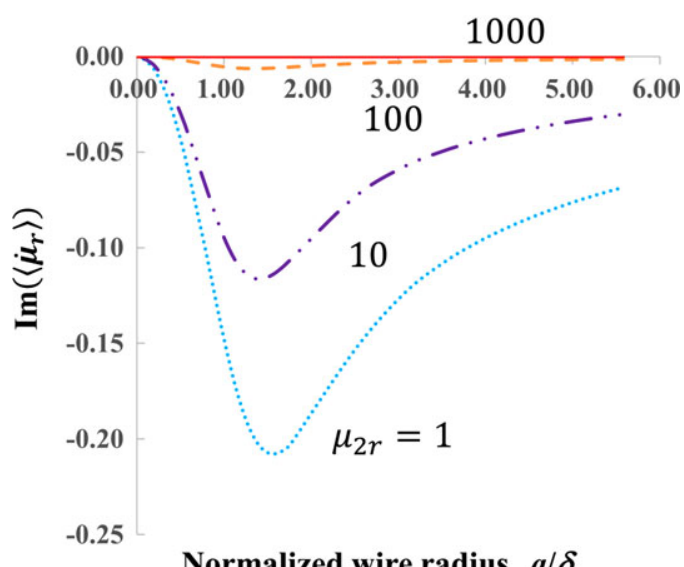

(b) Imaginary part

F I G U R E 5 Dependence of homogenized relative permeability of MP coil on normalized radius $a / \delta$ [Color figure can be viewed at wileyonlinelibrary.com]

TA B L E 2 Specification of MP coil shown in Figure 4

\begin{tabular}{llll}
\hline $\mathrm{a}$ & $0.37 \times 10^{-3}$ & $\eta$ & 0.64 \\
$\mathrm{~b}$ & $0.50 \times 10^{-3}$ & $f$ & $1.0 \sim 1.0 \times 10^{6}$ \\
$\mathrm{c}$ & $5.76 \times 10^{7}$ & $\mu_{1 \mathrm{r}}$ & 1.0 \\
\hline
\end{tabular}

acteristic of $\left\langle\dot{\mu}_{r}\right\rangle$ obtained from Equations (23) and (26) using the coil's filling factor $\eta$. Analysis specifications are given in Table 2 . In the graphs, $a / \delta$ on the horizontal axis is normalized radius (nondimensional value); skin effect becomes pronounced with $a / \delta \geq 1$. As indicated by the results of Figure 5, real part of $\left\langle\dot{\mu}_{r}\right\rangle$ expressing relative permeability grows, while its imaginary part expressing loss decreases, as relative permeability of magnetic plating is increased.

\section{2 | Effective impedance and magnetic flux lines in MP coil}

We analyze impedance of MP coil in Figure 4 by the proposed method. Here we assume axial symmetry and apply 


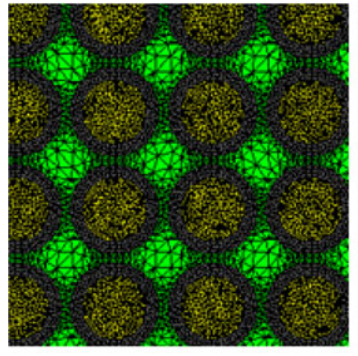

(a) Conventional FEM

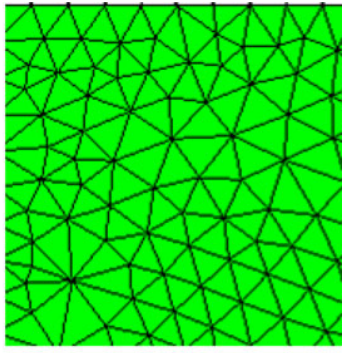

(b) Proposed method
F I G U R E 6 Finite elements in MP coil region [Color figure can be viewed at wileyonlinelibrary.com]

quasi-static electromagnetic field FEM using $\langle\dot{\mu}\rangle$ (proposed method). For comparison, we also use quasi-static electromagnetic field FEM without homogenization (conventional method). In case of the conventional method, skin and proximity effects must be considered in element division. Thus, we set elements in MP coil region as shown in Figure 6(a) so that the total number of elements in that region is 83572 . On the other hand, with the proposed method, MP coil region is homogenized; as a result, element division is as shown in Figure 6(b), the total number of elements in MP coil region being 728 . Figure 7 shows impedance frequency characteristics for this element division with relative permeability of plating varied at $\mu_{2 r}=1,10,100,1000$. As can be seen from Figure 7, the proposed method agrees well with the conventional method. Almost same results were obtained with the proposed method, even though the number of elements in coil was reduced to about $1 / 100$. A difference in results between the conventional FEM and the proposed method can be explained, for example, by the following reasons: (1) A uniform magnetic field is assumed in the proposed method, though actually there exists some nonuniformity; (2) sufficiently small elements were used in the conventional FEM though some numeric errors remained because magnetic plating error was thin.

Above we considered a 2D problem; in case of 3D analysis, the conventional method requires solving equations with enormous number of unknowns, and usefulness of the proposed method becomes even more pronounced. Next, we compare magnetic flux lines around MP coil assuming $f=1 \mathrm{MHz}$, $\mu_{2 r}=100.0$. The analysis results are presented in Figure 8. As can be seen from the diagram, magnetic flux distributions obtained by the two methods are almost same.

\section{3 | Relative permeability of magnetic plating and impedance characteristics}

Next, we consider dependence of coil impedance in Figure 4 on relative permeability $\mu_{2 r}$ of magnetic coating. We computed impedance of MP coil with driving frequency $f$ of input current fixed at $1 \mathrm{MHz}$, while varying $\mu_{2 r}$ in the range of 11000 . With $\mu_{2 r}=1$, magnetic plating is equivalent to conven-

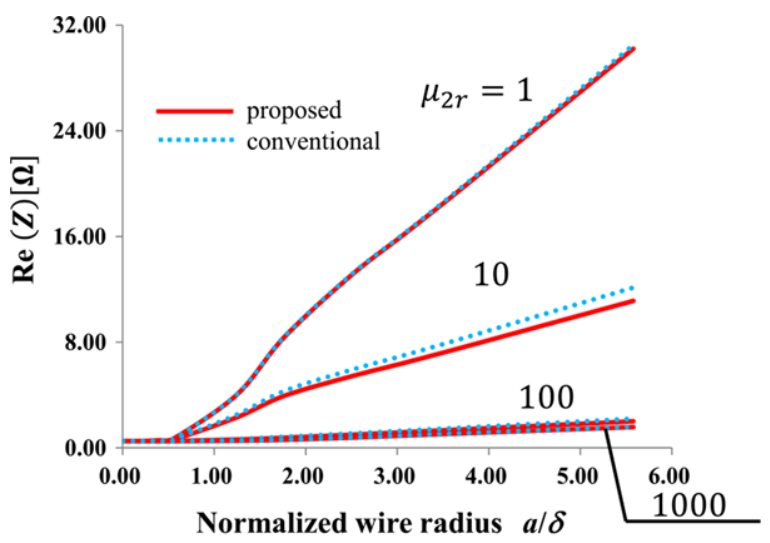

(a) Real part

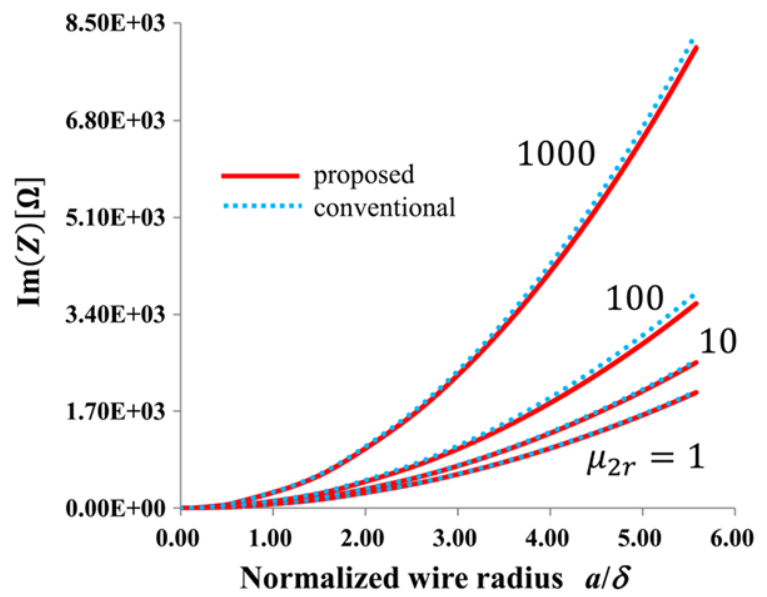

(b) Imaginary part

F I G U R E 7 Impedance of MP coil versus $a / \delta$ [Color figure can be viewed at wileyonlinelibrary.com]

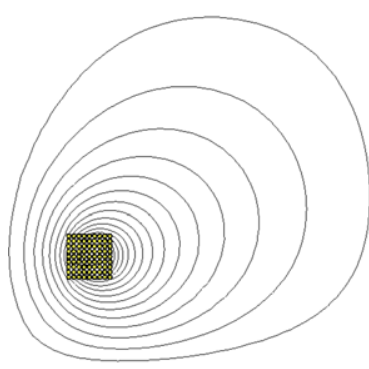

(a) Conventional FEM

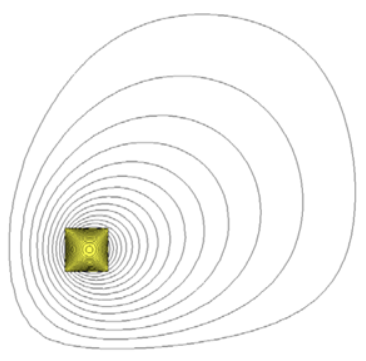

(b) Proposed method
F I G U R E 8 Comparison of magnetic fluxes [Color figure can be viewed at wileyonlinelibrary.com]

tional insulating coating, and MP coil impedance is equivalent to that of copper wire without magnetic plating. The analysis results are shown in Figure 9. As can be seen from the diagram, real part of impedance expressing loss decreases with higher $\mu_{2 r}$, while imaginary part increases. This means that loss caused by proximity effect can be reduced due to magnetic shielding. Therefore, MP coil offers considerable reduction of ac resistance as compared to conventional copper wire model; on the contrary, inductance can be increased. 


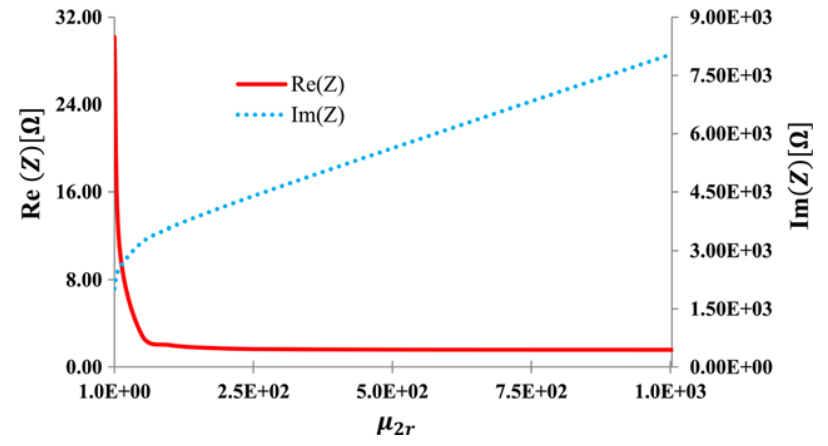

F I G U R E 9 Dependence of impedance on $\mu_{2 r}$ [Color figure can be viewed at wileyonlinelibrary.com]

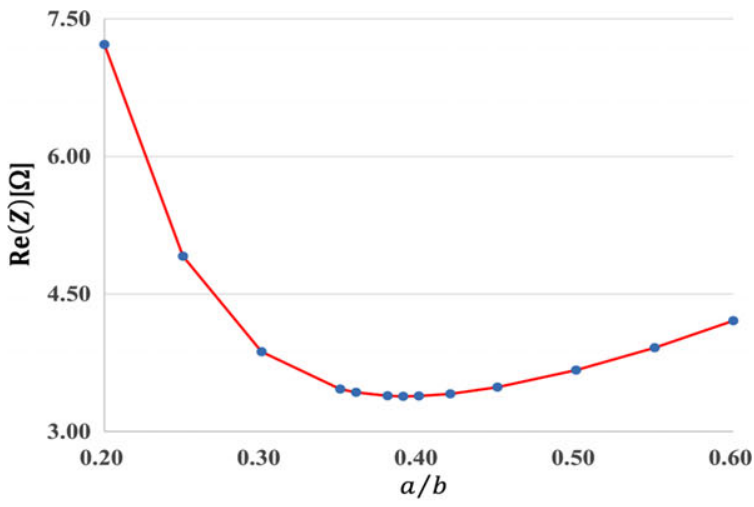

(a) $\mu_{2 r}=10$

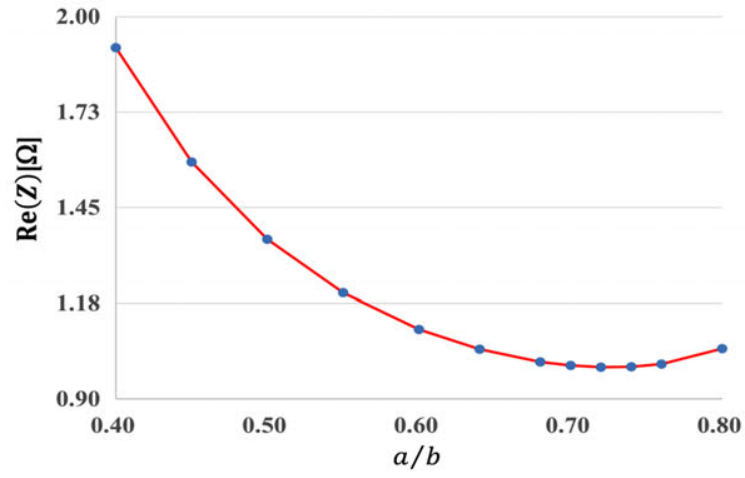

(b) $\mu_{2 r}=100$

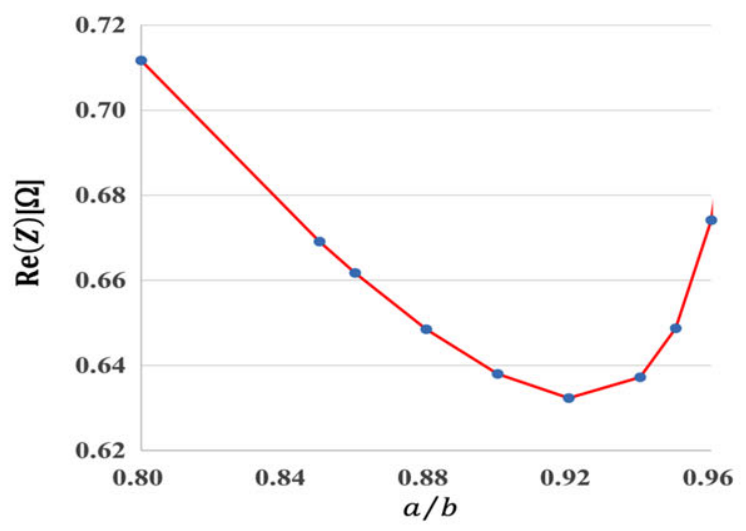

(c) $\mu_{2 r}=1000$

F I G URE 10 Dependence of $R_{\mathrm{ac}}$ on normalized wire radius $a / b$ [Color figure can be viewed at wileyonlinelibrary.com]

\subsection{Optimization of MP coil structure}

When copper wire radius $a$ is increased at a fixed MP coil radius $b$, dc resistance decreases while plating becomes thinner, and ac resistance grows. Thus, we consider relationship between copper wire radius $a$ and loss. For this purpose, we fixed driving frequency $f$ of input current at $200 \mathrm{kHz}$, and examined how MP coil impedance varies with wire radius $a$ of $0.05-0.50 \mathrm{~mm}$. Thus obtained results at relative permeability of plating $\mu_{2 r}=1,10,100,1000$ are shown in Figure 10. As can be seen from the diagram, the wire radius $a$ to minimize ac resistance depends on $\mu_{2 r}$. When $\mu_{2 r}$ is low, thick magnetic plating is needed to minimize loss. On the contrary, the plating layer can be made thinner when $\mu_{2 r}$ is high. In our case, minimum loss in MP coil is achieved around $a / b=0.39$ at $\mu_{2 r}=10$, around $a / b=0.72$ at $\mu_{2 r}=100$, and around $a / b=0.92$ at $\mu_{2 r}=1000$.

\section{4 | CONCLUSION}

In this article, we proposed homogenization of MP coil using macro effective relative permeability as well as a FE method based on such homogenization. With the proposed method, MP coil region can be treated as a uniform medium with macro complex effective permeability, which makes possible analysis with a rough element division. Homogenized analysis of MP coil impedance offered good agreement with conventional FE analysis. Besides, magnetic flux distributions almost coincided as well. In addition, we quantitatively clarified relationship between relative permeability of magnetic plating in MP coil and coil's ac resistance and inductance. We also discussed optimization of MP coil design. The proposed method can be applied to 3D analysis, too. Future topics include analysis in case of multi-layer magnetic plating and conductive magnetic plating or twisted MPW, application to actual electric devices for contactless power transfer, etc.

\section{REFERENCES}

1. Chai Y, Yamamoto T, Watanabe, S, et al. Examination of LLC Resonant Converter using magnetoplated wire. The Papers of Technical Meeting, IEE Japan, MAG-16-49, LD-16-41; 2016:57-62.

2. Yamamoto T, Sugimura K, Sato T, et al. Examination of LLC Resonant Converter using magnetoplated wire and carbonyl-iron powder/epoxy composite bulk magnetic core. The Papers of Technical Meeting, IEE Japan, MAG-16-50, LD-16-42; 2016:63-68.

3. Mizuno B, Yamamoto D, Iida K, et al. AC resistance analysis of transformer using magnetoplated wire. The Papers of Technical Meeting, IEE Japan, SA10-28; 2010. (in Japanese)

4. Mizuno T, Suzuki T, Asahina, T, et al. Reduction in eddy current loss in conductor using magnetoplated wire. IEEJ Trans FM. 2007;127(10):611-620. (in Japanese) 
5. Mizuno T, Enoki S, Asahina T, et al. Linearity range of an eddy current displacement sensor in relation to the FE film thickness of magnetoplated wire. J Magn Soc Jpn. 2007;31:103-108.

6. Igarashi $\mathrm{H}$, Sato $\mathrm{Y}$, Ito $\mathrm{Y}$. Macroscopic magnetic properties considering eddy currents: analysis of fine conductive lines. IEEJ. 2015. SA-15-032, RM-15-024;2015:111-115.

7. Igarashi H. Semi-analytical approach for finite element analysis of multi-turn coil considering skin and proximity effects. IEEE Trans Magn. 2017;53(1):7400107.

8. Ollendorff F. Magnetstatik der Massekerne. Arch f Electrotechnik. 1931;25:436-447.

9. Landau LD, Pitaevskii LP, Lifshitz EM. Electrodynamics of Continuous Media. 2nd ed. Oxford, England: Butterworth-Heinemann; 1984.

10. Ferreira JA. Improved analytical modeling of conductive losses in magnetic components. IEEE Trans Power Electron. 1994;9(1):127131.

\section{AUTHORS ' BIOGRAPHIES}

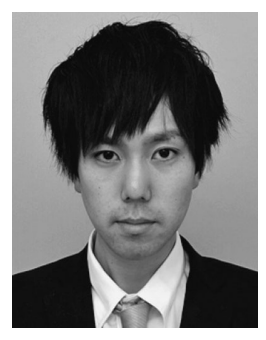

S. Fujita, non-member, born 1991: In 2015 he graduated from Hokkaido University. He is now pursuing postgraduate studies at the University (Graduate School of Information Science and Technology, Division of Systems Science and Informatics). He is involved in FEM analysis of litz wire, eddy-current displacement sensors, and numerical electromagnetic field analysis of contactless power supply systems.

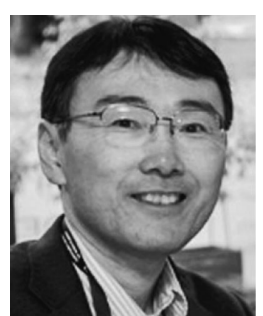

H. Igarashi, member: In 1984 he completed postgraduate studies at Hokkaido University (Graduate School of Engineering). Since 2004 he is a professor at Hokkaido University (Graduate School of Information Science and Technology). From 1995 to 1997 he was a visiting researcher at Technical University of Berlin. He received doctorate in engineering research in electromagnetic field analysis, optimization, and energy harvesting. He is a board member of International COMPUMAG Society, JASCOME, and JSAEM. In 2016 he received IEEJ Distinguished Paper Award and commended for Science and Technology by the Minister of Education, Culture, Sports, Science and Technology. He has membership of JASCOME, JSAEM, IEEE, IEICE, and JSST.

How to cite this article: Fujita S, Igarashi $\mathrm{H}$. Finite element analysis of multi-turn magnetoplated coils using homogenization method. Electr Eng Jpn. 2018;1-9. https://doi.org/10.1002/eej.23156 